\title{
FERMIONIC QUANTUM SPIN GLASS TRANSITIONS
}

\author{
Reinhold Oppermann and Bernd Rosenow \\ Institut für Theoretische Physik, Universität Würzburg, D-97074 Würzburg
}

\begin{abstract}
This article reviews recent progress of the analytical theory of quantum spin glasses (QSG). Exact results for infinite range and one loop renormalisation group calculations for finite range models of either insulating or metallic type are presented. We describe characteristics of fermionic spin glass transitions and of fermionic correlations which are affected by these transitions and by spin glass order. Connections between tricritical thermal- and $T=0$ QSG transitions are described. A general phase diagram with tricritical QSG transitions caused either by random chemical potential or by elastic electron scattering, and implying discontinuous $T=0$-transitions in weak and in strong filling regimes, is also derived.
\end{abstract}

\section{Introduction}

Fermionic quantum spin glasses form a part of the overlap regime of interacting disordered fermion systems and spin glasses. Understanding such systems with frustrated or with general random interactions between fermionic spins touches many currently active research fields. The wellknown transverse field Ising spin glass can also be represented as a pseudofermionic quantum spin glass with imaginary chemical potential and thus be treated analogously to the ones discussed here which involve real fermion spins. One of the attractive features of fermionic spin glasses is the connection of spin and charge degrees of freedom. Even in a strongly localized Ising limit (with all spin and charge variables commuting) quantum statistics remains indispensable, since it governs the relative occupation of magnetic and nonmagnetic states. This provides a link between spin- and charge correlations, exerting a major influence on magnetic phase diagrams for example. Nonanalytic interplay is observed in the simplest fermionic spin glass, the SK-model extended to a four-state-per-site fermionic space $\left(I S G_{f}\right)$. This phenomenon occurs at a thermal tricritical point, separating continuous spin glass transitions around half-filling from discontinuous regimes at either strong or weak filling. This structure of the phase diagram is compared with a similar one of classical spin 1 models including the clean BEG-model. Furthermore, model definitions on fermionic space generate a large set of correlation functions which display ubiquitous quantum dynamics of these models on the fermionic level, ie beyond the one seen in spin- and charge-correlations and caused by electronic transport. The fermionic Green's function and its density of states content is one of those quantities which probe the commutation properties of single 
fermion operators with the hamiltonian. The $I S G_{f}$ shows such quantumdynamics. Moreover, correlations of the statistical density of states fluctuations turn out to be related to the spin glass order parameter, which introduces Parisi replica symmetry breaking into the charge sector.

\section{The Grand Canonical Fermionic Ising Spin Glass}

In contrast to standard spin glass models like SK- or transverse field models, the models of interacting true fermion spins are naturally described in the grand canonical ensemble. Statistically fluctuating spin interactions leave only the choice between nonrandom local fermion concentration or nonrandom chemical potential. In any case however an effective spin dilution due to thermal redistribution among magnetic and nonmagnetic states is controlled by either $\mu$ or $\nu$. This form of thermal spin dilution comprises at a special point the case of nonanalytically communicating chargeand spin-fluctuations. The simplest model displaying this type of behavior is the Ising spin lass on a fermionic space $\left(I S G_{f}\right)$ with four states per site, defined by the hamiltonian $H=-\frac{1}{2} \sum_{i \neq j} J_{i j} \sigma_{i} \sigma_{j}-\mu \sum_{i} n_{i}$ with spins $\sigma_{i}=\Psi_{i, \alpha}^{\dagger} \sigma_{\alpha \beta}^{z} \Psi_{i, \beta}$, particle number operator $n_{i}=\Psi_{i, \alpha}^{\dagger} \Psi_{i, \alpha}$ and gaussian distributed exchange integrals $J_{i j}$ [1]. The fermionic field operators obey the usual commutator relations $\left\{\Psi_{i \alpha}, \Psi_{j \beta}\right\}=0$ and $\left\{\Psi_{i \alpha}^{\dagger}, \Psi_{j \beta}\right\}=\delta_{i j} \delta_{\alpha \beta}$. Expressing the partition function with the help of Grassman integrals the disorder averaging of the free energy is performed by means of the replica trick $\beta[F]_{a v}=[\log Z]_{a v}=\lim _{n \rightarrow 0} \frac{1}{n}\left(1-\left[\Pi_{\alpha=1}^{n} Z^{\alpha}\right]_{a v}\right)$. As a guide to the global phase diagram we study an exactly solvable infinite range version of the model but the formulae obtained in this subsection may equally well be considered as saddle point approximation for an interaction with finite range.

\subsection{Phase Diagram}

Details of the calculation are given in [1], here we just state the result for the replica symmetry broken saddle point free energy

$$
\begin{aligned}
\beta f= & \frac{1}{4} \beta^{2} J^{2}\left[(1-\tilde{q})^{2}-\left(1-q_{1}\right)^{2}+q_{1}^{2}-\int_{0}^{1} d x q^{2}(x)\right]-\ln 2-\beta \mu \\
& -\lim _{K \rightarrow \infty} \int_{z_{K+1}}^{G} \ln \left[\int _ { z _ { K } } ^ { G } \left[\ldots \left[\int_{z_{1}}^{G}(\cosh (\beta \tilde{H})\right.\right.\right. \\
& \left.\left.\left.\left.+\cosh (\beta \mu) \exp \left(-\frac{1}{2} \beta^{2} J^{2}\left(\tilde{q}-q_{1}\right)\right)\right)^{m_{1}}\right]^{m_{2} / m_{1}} \cdots\right]^{m_{k} / m_{K-1}}\right]^{1 / m_{K}}
\end{aligned}
$$

Notice the appearance of the Parisi variables $q_{\nu}$ and the additional $\tilde{q}$ which lies at the heart of the following discussion. The Edwards - Anderson order 
parameter $q_{E A}=\lim _{t \rightarrow \infty}<S_{i}(t) S_{i}(0)>$ describes the freezing of spins in the spin glass phase and is given by the Plateau height $q(1)$ of the order parameter function, whereas the replica diagonal $\tilde{q}=\left[\left\langle\sigma^{a} \sigma^{a}\right\rangle\right]_{a v}$ is related to the average filling factor $[\nu]_{a v}=\frac{1}{N} \sum_{i}\left[n_{i}\right]_{a v}$ by $[\nu]_{a v}=1+\tanh (\beta \mu)(1-\tilde{q})$. The last relation is exact even in the case of replica symmetry breaking. To obtain information about the phase diagram a replica symmetric approximation is sufficient, though. The symmetric saddle-point solutions are $q=\int_{z}^{G} \frac{\sinh ^{2}[\beta \tilde{H}(z)]}{\mathcal{C}_{\mu}^{2}(z)}$ and $\tilde{q}=\int_{z}^{G} \frac{\cosh [\beta \tilde{H}(z)]}{\mathcal{C}_{\mu}(z)}$ with $\mathcal{C}_{\mu}(z)=\cosh [\beta \tilde{H}(z)]+\cosh (\beta \mu) \exp \left[-1 / 2 \beta^{2}(\tilde{q}-\right.$ $q)$. Phase transitions are signalized by vanishing masses of the order parameter propagators which in the saddle point formalism are given by second derivatives of the free energy. Hence a positive mass for $\tilde{q}$ and a negative one for $q$ guarantees stability. A similar system of coupled stability conditions was found for the BEG - model [2] and for a SK - model with crystal field [3]. Analyzing the stability limits one obtains curves of critical spin and charge fluctuations, respectively:

$$
\begin{gathered}
\mu_{c 1}(T)=T \cosh ^{-1}[(1 / T-1) \exp [1 /(2 T)]] \\
\mu_{c 2}(T)=T \cosh ^{-1}\left[\frac{\left(1 \mp \sqrt{1-8 T^{2}}\right)^{2}}{8 T^{2}} \exp \left[\frac{2}{1 \mp \sqrt{1-8 T^{2}}}\right]\right]
\end{gathered}
$$

The two curves have a common tangent point at $\mu_{c 3}=1 / 3 \cosh ^{-1}[2 \exp (3 / 2)]=$ $0.961056, T_{c 3}=1 / 3$. For smaller values of the chemical potential the paramagnetic solution becomes unstable to spin fluctuations first, at the common tangent point both types of fluctuations become critical simultaneously and tricritical behaviour results.

\subsection{Tricritical Point TCP: Exponents and Special Features}

An expansion of the saddle point equations around the tricritical point yields in leading order

$$
0=g r_{g}-r_{T} \delta T^{2}+6 \delta T \delta \tilde{q}-\frac{3}{4} \delta \tilde{q}^{2}+3 q^{2}, \quad 0=6 q(\delta \tilde{q}-\delta T-q)
$$

where $\delta \tilde{q} \equiv \tilde{q}-\tilde{q}_{T C P}, g J=\mu-\mu_{c 3}+\left(\zeta^{-1} J-\mu_{c 3}\right) 3 \delta T$ as nonordering field, and $\delta T \equiv T-T_{c 3}$. The constants are given by $r_{g}=\frac{2 \zeta}{3}, r_{T}=2\left(1-\frac{3}{4} \zeta^{-2}\right)$ with $\zeta \equiv \tanh \left(\mu_{c 3} / T_{c 3}\right) \simeq 0.9938$. The average filling factor corresponding to $\mu_{c 3}$ is evaluated as $\left[\nu_{c 3}\right]_{a v} \simeq 1.6625$. From (4) we get for $\mathrm{q}=0$

$$
\delta \tilde{q}_{d i s}=4\left(\delta T \pm|\delta T| \sqrt{1+\frac{r_{g} g}{12 \delta T^{2}}-\frac{r_{T}}{12}}=4(\delta T \pm|\delta T| W)\right.
$$

Only the solution with the - sign corresponds to a minimum of the free energy, in a region close to the line $\delta \mu=-3\left(1 / \zeta-\mu_{c 3}\right) \delta T=-0.1354 \delta T$ (tangent 
to both $\mu_{c 1}\left(T_{c 3}\right)$ and $\left.\mu_{c 2}\left(T_{c 3}\right)\right) \mathrm{g}$ is of order $\delta T^{2}$ or smaller and usual critical behaviour results. However, if $\mathrm{g}$ is of order $\delta T$ or larger the solution becomes to leading order $\delta \tilde{q}=\sqrt{\frac{r_{g} g}{12}}$ and thus displays a nonanalytical dependence on temperature and / or chemical potential. This type of crossover can also be seen from the scaling form of the free energy $f_{\text {dis }}=|\delta T|^{2-\alpha} \mathcal{G}\left(\frac{g}{\delta T^{2}}\right)$ which allows for the identification of the specific heat exponent $\alpha=-1$ and the crossover exponent $\phi=2$. The crossover function $\mathcal{G}(x)$ is regular for small $\mathrm{x}$ and has the asymptotic form $\mathcal{G}(x) \underset{x \rightarrow \infty}{\approx}\left(\frac{g}{\delta T^{2}}\right)^{\frac{2-\alpha}{\phi}}\left(\mathcal{G}_{\infty}+\right.$ regular corrections). In the tricritical region the leading singularity in the free energy is given by $f_{T C P, s i n g}=\frac{4}{\sqrt{3}}\left(r_{g} g\right)^{\frac{3}{2}}$. For $\delta \mu=0$ we have $g \sim \delta T$ and can read off the tricritical specific heat exponent $\alpha_{3}=\frac{1}{2}$ from above.

Using $q=\delta \tilde{q}-\delta T$ one finds ordered solutions displaying the same crossover as discussed above, the transition from paramagnet to spin glass being continous for positive $\delta T$ and first order for negative temperature deviations. In the tricritical regime we find $q=\delta \tilde{q}=\frac{2}{3} \sqrt{-r_{g} g}$ which yields the tricritical order parameter exponent $\beta_{3}=\frac{1}{2}$ and suggests that $q$ and $\delta \tilde{q}$ act as order parameters simultaneously. From the fluctuation Lagrangian (see section 3 ) one reads off mass squared proportional to $\delta T$ and hence $\gamma_{3}=\beta_{3}=\alpha_{3}=\frac{1}{2}$.

\section{Tricritical Landau Theory and the Parisi Solution of the Fermionic Ising Spin Glass}

We derived a fluctuation theory for the tricritical and finite range $I S G_{f}$; for $T \neq 0$ a Lagrangian of the same structure is obtained for models including transport mechanism by integrating out dynamical degrees of freedom

$$
\begin{aligned}
L & =\frac{1}{t} \int d^{d} x\left[-\frac{3 h^{2}}{2 J} \sum Q^{a b}+\frac{r \kappa_{1}}{\left(\kappa_{2}\right)^{2}} \sum Q^{a a}+\frac{1}{2} \sum Q^{a a}\left(-\nabla^{2}+u\right) Q^{a a}\right. \\
& +\frac{1}{2} \operatorname{Tr}^{\prime}\left(\nabla Q^{a b}\right)^{2}-\frac{1}{t} \sum^{\prime} Q^{a a} Q^{b b}-\frac{\kappa_{1}}{3} \sum\left(Q^{a a}\right)^{3} \\
& \left.-\frac{\kappa_{3}}{3} \operatorname{Tr}^{\prime} Q^{3}-\kappa_{2} \sum Q^{a a} Q^{a b} Q^{b a}+\frac{y_{4}}{4} \sum^{\prime}\left(Q^{a b}\right)^{4}\right],
\end{aligned}
$$

Here $4\left(\frac{\kappa_{1}}{t}\right)^{(0)}=\left(\frac{\kappa_{2}}{t}\right)^{(0)}=\left(\frac{\kappa_{3}}{t}\right)^{(0)}=\frac{3^{3}}{2}$ and $u^{(0)}=0$ denote the bare coefficients at tricriticality. One fourth order term relevant for replica symmetry breaking is kept. Replicas under $\sum^{\prime}$ or $T r^{\prime}$ are distinct. The $Q^{a a} Q^{b b}$-coupling is renormalization group generated as in the metallic quantum spin glass, its effects will be discussed in a subsequent section. 
Fig. 1.

Vicinity of the tricritical point (TCP) for positive

FERMIONIC QUANTUM SPIN GLASS cTRAdedPIfotential. Con-

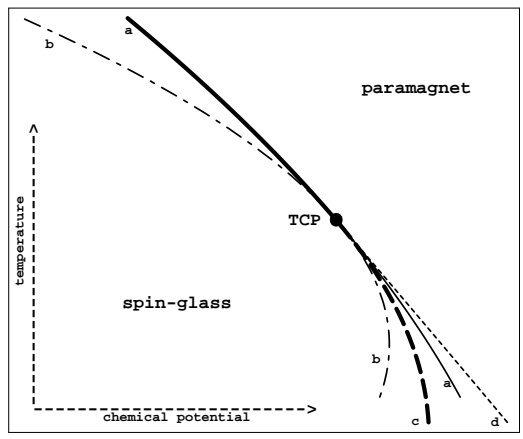

tinuous spin glass transitions occur on curve (a) above the TCP (thick unbroken line). Below the TCP first order thermodynamic transitions take place on curve (c). Curve (d), starting at the TCP, and curve (b) below the TCP limit the existence regime of ordered and disordered phases respectively.

Inserting the Parisi Ansatz for replica symmetrie breaking in the Lagrangian (6) the expansion of the replica symmetry broken saddle point free energy around the tricritical point reads

$$
\begin{aligned}
f & -f_{T C P}=\mu-\mu_{c 3}-\frac{3 h^{2}}{2 J}\left[\delta \tilde{q}-\int_{0}^{1} q(x) d x\right]+J\left\{\left(\frac{3}{2} r_{g} g-r_{\tau} \tau^{2}\right) \delta \tilde{q}\right. \\
& +\frac{3}{2} \tau\left[(\delta \tilde{q})^{2}-\int_{0}^{1} d x q^{2}(x)\right]-\frac{3}{2}\left[\int_{0}^{1} d x\left[x q^{3}(x)+3 q(x) \int_{0}^{x} d y q^{2}(y)\right]\right. \\
& \left.\left.-3 \delta \tilde{q} \int_{0}^{1} d x q^{2}(x)+\frac{1}{4}(\delta \tilde{q})^{3}\right]-\frac{y_{4}}{4} \int_{0}^{1} d x q^{4}(x)\right\}
\end{aligned}
$$

Here variables and constants are defined as in (3), $\tau=\delta T / T_{c 3}$. In contrast to crystal-field split spin glasses [3] the quartic coefficient $y_{4}$ of our free energy, Eq.(7), is nonzero and one obtains the Parisi solution $q(x)=\frac{9}{2 y_{4}} x$ for $0 \leq x \leq x_{1}$ and $q(x)=q(1)$ for $x_{1} \leq x \leq 1$. The plateau height is found to satisfy $q(1)=\delta \tilde{q}+O\left(\delta \tilde{q}^{2}\right)$. Consequently, plateau and breakpoint scale like $\sqrt{|\tau|}+O(\tau)$ at the $T C P$, while linear $\tau$-dependence is reserved to $T_{c}>T_{c 3}$. Adapting the notation of [4] we express our result for the irreversible response $q(1)-\int_{0}^{1} q(x) \sim|\tau|^{\beta_{\Delta}}$ in terms of the exponent $\beta_{\Delta 3}=1$ for $T \rightarrow T_{c 3}$ and $\beta_{\Delta}=2$ for $T \rightarrow T_{c}>T_{c 3}$. For the Almeida-Thouless line at tricriticality we find $\frac{H^{2}}{J^{2}}=\frac{80}{81}\left(\frac{2}{3}\left(1-\frac{\mu_{c 3}}{J} \tanh \left(\frac{3 \mu_{c 3}}{J}\right)\right)\right)^{3 / 2} \tau_{A T}^{3 / 2}+O\left(\tau_{A T}^{2}\right)$ with $\tau_{\mathrm{AT}} \equiv \frac{T_{c 3}-T_{A T}(H)}{T_{c 3}}$. Hence we obtain the critical exponent $\theta_{3}=\frac{4}{3}$ near $T_{c 3}$, while $\theta=\frac{2}{3}$ for all $T_{c}>T_{c 3}$. These values do not satisfy the scaling relation $\theta_{3}=\frac{2}{\beta_{3}}$ with $\beta_{\Delta_{3}}=1+\left(\gamma_{3}-\alpha_{3}\right) / 2$. Along the lines described in [4], this problem of mean-field exponents will be resolved below by the renormalization group analysis of the coupling $y_{4}$ of the finite-range and finite-dimensional ISG $G_{f}$.

\section{Replica Symmetry Breaking for Fermions}

The fermionic Ising spin glass allows for an exact evaluation of the bare fermion propagator $G_{i j, \sigma}=<<\frac{1}{i \epsilon_{n}+\mu+\tilde{H}}>>$ at least in the disordered phase; 
here $\tilde{H}$ denotes the usual effective field and the double average refers to the replica-local and the Parisi block decoupling fields. The result can be written in the form

$$
\begin{aligned}
G_{i j, \sigma}\left(\epsilon_{n}\right)= & -i \sqrt{\frac{\pi}{2 J \tilde{q}}} \sum_{\lambda=0, \pm 1} \frac{\left(2-\lambda^{2}\right) e^{\frac{1}{2}(\beta J \lambda)^{2} \tilde{q}} \operatorname{ch}\left(\left(1-\lambda^{2}\right) \beta \mu_{\sigma}\right)}{\exp \left(\frac{1}{2} \beta^{2} J^{2} \tilde{q}\right)+\operatorname{ch}\left(\beta \mu_{\sigma}\right)} \\
& \left(1-\operatorname{erfc}\left(\frac{\epsilon_{n}-i \mu_{\sigma}+i \lambda \beta J^{2} \tilde{q}}{J \sqrt{2 \tilde{q}} J}\right)\right) \exp \left(\frac{\epsilon_{n}-i \mu_{\sigma}+i \lambda \beta J^{2} \tilde{q}}{\sqrt{2 \tilde{q}} J}\right)^{2} \delta_{i j},
\end{aligned}
$$

where $\mu_{\sigma} \cong \mu+\sigma H$ includes a magnetic field $H$ and $\epsilon_{n}=(2 n+1) \pi k_{B} T / \hbar$. One easily extracts the disorder averaged electronic density of states [5]

$$
<\rho(\epsilon)>=\frac{1}{\sqrt{2 \pi \tilde{q} J}} e^{-\frac{(\epsilon+\mu)^{2}}{2 J^{2} \tilde{q}}} \frac{\operatorname{ch}(\beta \mu)+\operatorname{ch}(\beta(\epsilon+\mu))}{\operatorname{ch}(\beta \mu)+\operatorname{ch}(\beta H) e^{\frac{1}{2} \beta^{2} J^{2} \tilde{q}}}
$$

Below the freezing temperature $O\left(q^{2}\right)$-corrections occur in $\langle\rho\rangle$. Statistical fluctuations $\delta \rho=\rho-\langle\rho\rangle$ of the density of states are for example observable in $\left\langle\delta \rho_{\sigma}^{a}(\epsilon) \delta \rho_{\sigma^{\prime}}^{a^{\prime}}\left(\epsilon^{\prime}\right)>\right.$. Taking Parisi symmetry breaking into account, this correlation becomes a function of the Parisi parameter x. Picking the one with $\sigma=\sigma^{\prime}$ and $\epsilon=\epsilon^{\prime}$ we obtain the (zero field) result

$$
\begin{gathered}
<\delta \rho_{\sigma}(\epsilon) \delta \rho_{\sigma}(\epsilon)>(x)=\frac{1}{4 \pi J^{2} \tilde{q}_{c}^{5}}\left(1-\tilde{q}_{c}+\tilde{q}_{c} e^{-\frac{1}{2 \tilde{q}_{c}}} \cosh \left(\frac{\epsilon+\mu}{J \tilde{q}_{c}}\right)\right)^{2} \\
\left(1+\tilde{q}_{c}-\frac{(\epsilon+\mu)^{2}}{J^{2}}\right)^{2} e^{-\frac{(\epsilon+\mu)^{2}}{J^{2} \tilde{q}_{c}}} q^{2}(x)+O\left(q^{3}(x)\right) .
\end{gathered}
$$

This result shows that the fermionic density fluctuations reflect the irreversible magnetic response introduced by the spin glass order. It is valid for all fillings, one only has to insert the appropriate Parisi function and the filling-dependent value of the spin autocorrelation $\tilde{q}_{c}$ at the critical point; this implies that the scaling of the density correlator with $T-T_{c}$ is different in the second order regime (quadratic) and at the tricritical point (linear), while for the discontinuous regime a one-step RSB is expected. It is also clear that for models with transport mechanism the calculation of conductance fluctuations is of great interest. So far we have put aside the question of replica symmetry breaking of fermion propagators: by this we mean the possibility of a nonvanishing propagator between different replicas, ie $\left\langle\bar{\psi}^{a} \psi^{b \neq a}\right\rangle$. In the light of the Mezard-Parisi instability of the random field Ising model we feel that this preferably would occur as a fluctuation effect, if at all.

\section{$5 \quad$ Related Fermionic Spin Models}

\subsection{The Fermionic Ising Chain}

Similarities between the phase diagrams of the clean BEG-model and of the fermionic Ising spin glass can be taken as indicative for the fact that 
rather spin dilution than disorder is the source of the tricritical crossover from continuous to discontinuous phase transitions. While it is complicated to solve 1D fermionic Ising spin glasses exactly, the clean fermionic Ising chain offers some simple exact solutions. Here we shall provide insight into the role of the chemical potential and moreover generalize known results into the complex $\mu$-plane. Lee and Yang [7] derived the distribution of zeroes of the partition function of finite and infinite Ising chains within the complex magnetic field plane. Stimulated by the representation of conventional Ising chains by fermionic ones with special imaginary chemical potential, one may wish to extend the Yang Lee analysis to a fourdimensional space of complex $(\mu, \nu)$. The transfer matrix $\mathbf{T}_{\mathbf{f}}$ of the fermionic Ising chain reads

$$
T_{f}=e^{\beta \mu}\left(\begin{array}{cccc}
e^{\beta \mu} & 1 & e^{\frac{1}{2} \beta(\mu+h)} & e^{\frac{1}{2} \beta(\mu-h)} \\
1 & e^{-\beta \mu} & e^{\frac{1}{2} \beta(h-\mu)} & e^{-\frac{1}{2} \beta(\mu+h)} \\
e^{\frac{1}{2} \beta(\mu+h)} & e^{\frac{1}{2} \beta(h-\mu)} & e^{\beta(J+h)} & e^{-\beta J} \\
e^{\frac{1}{2} \beta(\mu-h)} & e^{-\frac{1}{2} \beta(\mu+h)} & e^{-\beta J} & e^{\beta(J-h)}
\end{array}\right)
$$

The transfer matrices $\mathbf{T}_{\mathbf{f}}$ and $\mathbf{T}_{\mathbf{s}}$ of the standard $S= \pm 1$-chain and their eigenvalues do not map onto each other at $\mu=i \frac{\pi}{2} T$, while the partition functions obey $Z_{f}^{(N)}=\operatorname{Tr} T_{f}^{N}=(2 i)^{N} Z_{s}^{(N)}\left(\mu=i \frac{\pi}{2} T\right)$ for any number $\mathrm{N}$ of sites. The largest eigenvalue determines the free energy of the infinite chain, while the second largest is required in addition to determine the correlation length. The eigenvalues for $h=0$ are found as

$$
\begin{aligned}
\lambda_{ \pm} & =e^{\beta \mu}\left[\operatorname{ch}(\beta \mu)+\operatorname{ch}(\beta J) \pm \sqrt{(\operatorname{ch}(\beta \mu)+\operatorname{ch}(\beta J))^{2}+4 \operatorname{ch}(\beta \mu)(1-\operatorname{ch}(\beta J))}\right] \\
\lambda_{0} & =0 \quad, \quad \lambda_{1}=2 e^{\beta \mu} \operatorname{sh}(\beta J) .
\end{aligned}
$$

The correlation length is given by $\xi=1 / \ln \left(\frac{\lambda_{+}}{\lambda_{1}}\right)$. In the $T \rightarrow 0$-limit a transition arises at $\mu=J$ and due to the properties

$$
\lambda_{1} \sim \exp (\beta(\mu+J)), \quad \lambda_{+} \sim\left\{\begin{array}{c}
\exp (\beta(\mu+J)), \quad \mu<J \\
\exp (2 \beta \mu), \quad \mu>J
\end{array}\right.
$$

Thus $\xi$ diverges only for $\mu<J$, since the energy for adding a fermion is larger than the gain from a magnetic bond if $\mu>J$. Hence

$$
\xi \sim\left\{\begin{array}{cl}
\exp (\beta(2 J-\mu)) & , 0<\mu<J \\
\exp (\beta J / 2) & , \mu=J
\end{array}\right.
$$

while $\xi \sim T /(\mu-J)$ for $\mu>J$. The filling factor shows for $T \rightarrow 0$ that the system is completely filled for $\mu>J$ (empty for $\mu<-J$ ). Thus there is no physical $T=0$-transition of this simple system. The correlation length diverges in the $T \rightarrow 0$ limit for all fillings $\nu$. The zero-field partition function shows that Yang-Lee zeroes approach $\mu= \pm J$ for $T \rightarrow 0$. This means that for $(h=0, T=0)$ nonanalytic behaviour (as a function of the real chemical 
potential) can only occur at the values $\mu= \pm J$. It is instructive to consider $N=2$ explicitly, which yields

$$
Z_{f}^{N=2}=4 e^{2 \beta \mu}\left[(\operatorname{ch}(\beta \mu)+\operatorname{ch}(\beta J))^{2}+\operatorname{ch}^{2}(\beta h)\left(e^{2 \beta J}-1\right)-\operatorname{sh}(2 \beta J)\right]
$$

This almost trivial case already shows zeroes at $\mu_{0}= \pm\left(J+\left(\frac{1}{2} \ln 2 \pm i \frac{\pi}{2}\right) T\right)$, while allowing for finite complex magnetic field the first zero different from $\pm J$ in the $T \rightarrow 0$-limit becomes possible with $\mu= \pm\left(i \frac{\pi}{4}+2 i m \pi\right) T$.More zeroes occur on the $T=0$-axis as $\mathrm{N}$ is increased. For $N \rightarrow \infty$ a density function is expected in accordance with $\xi$ diverging for any $\mu$.

\subsection{Mapping the twodimensional Ising model with imaginary magnetic field $h=\frac{i \pi}{2} T$ into fermionic space}

The complementary role of complex magnetic field and complex chemical potential can nicely be seen by recalling the exact solution for the $2 \mathrm{~d}$ Ising model $h=i \frac{1}{2} \pi T$. This value corresponds to $\mu=i \frac{1}{2} \pi T$, which maps the fermionic Ising model onto the one above. Thus the exact solution of the $2 \mathrm{~d}$ fermionic Ising model with $\mu=h=i \frac{1}{2} \pi T$ is known. Moreover this special model maps onto an interaction model of spinless fermions with a special species obeying bare Bose statistics but carrying along the minus signs of fermion interactions. The hamiltonian of this model can be written

$$
H=-\sum_{i j} J_{i j} \sigma_{i}^{z} \sigma_{j}^{z}-\mu \sum_{i}\left(n_{i \uparrow}+n_{i \downarrow}\right)-h \sum_{i} \sigma_{i}^{z}
$$

with $\mu=h=i \frac{\pi}{2} T$. This reduces to zero the imaginary field of one fermionic species, while the other field equals the distance between Bose- and FermiMatsubara energies. Setting $c=a_{\uparrow}$ and $d=a_{\downarrow}$ the hamiltonian reads

$$
H=-\sum_{i j} J_{i j}\left[c_{i}^{\dagger} c_{i} c_{j}^{\dagger} c_{j}-c_{i}^{\dagger} c_{i} d_{j}^{\dagger} d_{j}-d_{i}^{\dagger} d_{i} c_{j}^{\dagger} c_{j}+d_{i}^{\dagger} d_{i} d_{j}^{\dagger} d_{j}\right]-i \pi T \sum_{i} d_{i}^{\dagger} d_{i} .
$$

The new imaginary chemical potential $-i \pi T$ of $\mathrm{d}$-fermions renders their Matsubara energies and single particle statistics bosonic. Thus anticommuting $\mathrm{d}-$ bosons interact with c-fermions. All vertices retain the fermionic minus-signs. In the fermionic path integral the $\mathrm{c}-$ and $\mathrm{d}$-particles are described by the Grassmann fields $\psi_{c}(\tau)$ and by $\psi_{d}(\tau)$ respectively. The $i \pi T \hat{n}_{d}$-term can be absorbed by the phase transformation $\exp (i \pi \tau) \psi_{d}(\tau)=\tilde{\psi}_{d}(\tau)$. The new anticommuting fields $\tilde{\psi}_{d}$ obey unusual bosonic periodicity $\tilde{\psi}_{d}(\tau)=\tilde{\psi}_{d}(\tau+\beta)$ on the imaginary time axis, while $\psi_{c}(\tau)=-\psi_{c}(\tau+\beta)$ remains fermionic. The bosonic feature of the anticommuting fields $\psi_{d}$ 's is of course seen in the bare statistics. Perturbatively the above exact conclusion maps the $2 \mathrm{D}$ Ising model with $h=i \pi T / 2$ onto a coupled Fermi-Bose system (c-d) with additional (-1)-factors for each d-loop. 


\section{Metallic Quantum Spin Glass}

So far we have discussed the magnetic phase diagram of fermionic lattice gases having in mind that the tricritical phenomenon and universal quantities are not changed by coherent hopping of the electrons in flat bands. On the other hand it is well known [8], [9] that both a random chemical potential and an increasing band width suppress the transition temperature continuously down to zero thereby producing a quantum phase transition (QPT). The field theory of such a QPT

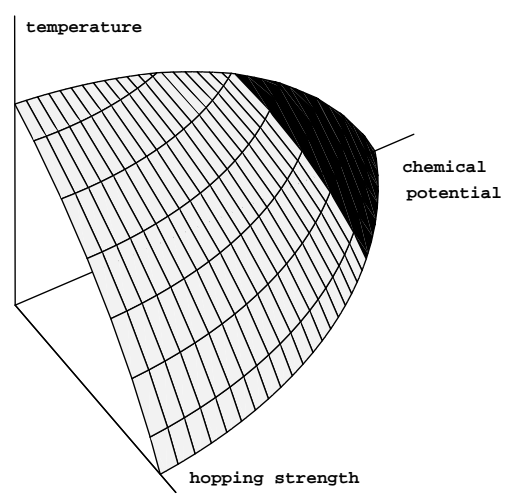

Fig. 2. Phase diagram of fermionic spin glasses as function of chemical potential, hopping, and temperature. Above the marked area the system is in the paramagnetic, below in the spin glass phase. The shaded area corresponds to continuous transition, the black area to first order transitions. Tricritical points are located at the boundary of the two regions.

shows surprising similarities with the thermal tricritical theory, above all the simultaneous occurence of critical charge and spin fluctuations [9]. However, electron hopping not only lowers the $T_{c}$ of 2 nd order transitions but also that of tricritical and 1st order transitions. For a metallic model with Gaussian random hopping and bandwidth $2 E_{0}$ we found a quantum TCP at

$$
E_{F}=\left(1-(5 / 8)^{\frac{1}{2}}\right)^{\frac{1}{2}} E_{0}, \quad J_{c}=3 \pi E_{0}\left[1-E_{F}^{2} / E_{0}^{2}\right]^{-\frac{3}{2}} / 32,
$$

Corrections to this Q - static approximation can be calculated by generalizing method [10]. The general phase diagram is sketched in figure 2 .

\section{Renormalisation Group Analysis}

\subsection{Tricritical Ising Spin Glass}

We performed a 1-loop RG calculation for tricritical fluctuations. At each RG step the mass of charge fluctuations $\delta Q^{a a}$ was shifted away. Introducing the anomalous dimensions $\tilde{\eta}$ and $\eta$ for diagonal and offdiagonal fluctuations one finds at one loop level the following $\mathrm{RG}$ relations $(\epsilon=8-d)$

$$
\frac{d r}{d l}=\left(\frac{d}{2}-11 \kappa_{1}^{2}+16 \kappa_{1} \kappa_{2}+6 \kappa_{2}^{2}\right) r-\kappa_{2}^{2}, \frac{d u}{d l}=2\left(1-\kappa_{1}^{2}\right) u-4 \kappa_{1}^{2}+4 \kappa_{1} \kappa_{2},
$$




$$
\frac{d \kappa_{1}}{d l}=\frac{\epsilon}{2} \kappa_{1}+9 \kappa_{1}^{3}, \frac{d \kappa_{2}}{d l}=\left(\frac{\epsilon}{2}+6 \kappa_{2}^{2}-\kappa_{1}^{2}+16 \kappa_{1} \kappa_{2}\right) \kappa_{2}, \frac{d \kappa_{3}}{d l}=\left(\frac{\epsilon}{2}+9 \kappa_{2}^{2}\right) \kappa_{3},
$$

Above $d=8$ the RG flows towards the Gaussian fixed point with mean field exponents, for $d<8$ there is no perturbatively accessible fixed point for real $\kappa^{\prime} s$. However, a preliminary analysis of the resulting strong coupling problem shows that there exists a solution with positive $\tilde{\eta}$ in contrast to the negative anomalous dimension typical of cubic field theories with imaginary coupling. The RG for the DIC $y_{4}$ showed that its long-distance behaviour is dominated by a $\kappa^{4}$-contribution (like in [4] but) for $d_{c}^{(u)}=8<d<10$. This leads to the modified MF exponent $\theta_{3}=8 /(d-4)$, which satisfies the scaling relation $\theta_{3}=2 / \beta_{\Delta_{3}}$ in $d_{c 3}^{(u)}=8$ and reduces to the MF-result in 10 dimensions. The dimensional shift by 2 in comparison with [4] is due to coupling $t$.

\subsection{Metallic Quantum Spin Glass}

In the theory of quantum phase transitions time-dependent fluctuations are treated on an equal footing with spatial fluctuations [9]. While for the Lagrangian (7) it was sufficient to consider only the $\omega=0$ - component of the $\mathrm{Q}$ - fields, in the quantum case all low energy fields must be kept as they are coupled via the quantum mechanical interaction $u \int d \tau\left(Q^{a a}\right)^{2}$. However, the value $z=4$ of the dynamical critical exponent renders the $\mathrm{u}$ - coupling dangerously irrelevant and allows for a perturbative mapping of the critical theory on a classical problem, the Pseudo - Yang - Lee edge singularity. This field theory has only one cubic coupling which corresponds to $\kappa_{1}$ in eq(7). The comparison of the metallic quantum case with the thermal tricritical theory allows one to understand the nature of the strong coupling RG fixed point: whereas the spin fluctuations in the thermal second order regime are governed by a perturbatively accessible fixed point, the TCP and the quantum case are charcterized by the combination of charge and spin fluctuations and a corresponding strong coupling problem.

\section{References}

[1] Oppermann R., Müller - Groeling A. (1993): Nucl. Phys. B401, 507

[2] Blume M., Emery V.J., Griffiths R.B. (1971): Phys. Rev. A 4, 1071

[3] Mottishaw P. J., Sherrington D. (1985): J. Phys. C 18, 5201

[4] Fisher D. S., Sompolinsky H. (1985): Phys. Rev. Lett. 54, 1063

[5] Rosenow B., Oppermann R. (1996): Phys. Rev. Lett. 77, 1608

[7] Lee T. D., Yang C. N. (1952): Phys. Rev. 87, 410

[8] Oppermann R., Binderberger M. (1994): Ann. Phys. 3, 494

[9] Sachdev S., Read N., Oppermann R, (1995): Phys. Rev. B 52, 10286

[10] Huse D., Miller J. (1993): Phys. Rev. Lett. 70, 3147 